\title{
The clinical criteria of brain death throughout the world: why has it come to this?
}

Eelco F. M. Wijdicks MD

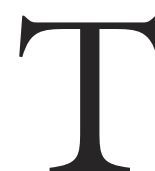

HE addition of neurological criteria of death - better known as brain death - to cardiorespiratory criteria of death was a paradigm shift which evolved when patients with acute brain injury could be resuscitated in emergency departments and intensive care units. Resuscitation of acute brain injury led to progression of devastating brain edema, shift, and eventually massive increase in intracranial pressure that would stop the blood flow at the entrance of the skull base. Multiple symposia were organized in Sweden, the United Kingdom, and Australia that tried to formulate brain death on the basis of neurological criteria. Brain death examination became a prerequisite to allow organ donation, and its concept has been fully accepted. Despite widespread acceptance of the criteria, there remained great variability in how brain death criteria were codified in different parts of the world. A review of the history of neurological death is presented by Baron et al. in this issue of the Journal. ${ }^{1}$

\section{The gold standard}

A seminal paper on neurological determination of brain death was written by the Harvard ad hoc Committee. $^{2}$ There was a desire in the 1960s to produce a brief but succinct document due to a pressing need in the critical care community for guidelines. To this day, the Harvard criteria remain an example of simplicity. The criteria were as follows:

1. Unreceptivity and unresponsiveness;

2. No movement or breathing;

3. No brainstem reflexes;

4. Flat electroencephalogram;

5. All tests repeated at least $24 \mathrm{hr}$ later, with no change, and exclusion of hypothermia (body temperature $<90^{\circ} \mathrm{F}$ or $32.2^{\circ} \mathrm{C}$ ) or central nervous system depressants.

\section{Brain death criteria throughout the world}

A recent survey explored the international practices for diagnosing brain death. ${ }^{3}$ Original brain death documents of 80 countries throughout the world were obtained. The differences in criteria were stunning. No differences appeared when the methods of examination of brainstem reflexes were compared; however, there were marked differences in how the apnea test was performed. The apnea test continued to concern physicians, although the procedure is simple and in most cases without complications. Testing the response with induced hypercarbia should remain standard if our goal is to document total brainstem destruction. Confirming apnea with a $\mathrm{PaCO}_{2}$ target value was used in only $59 \%$ of all guidelines. In others, preoxygenation with $100 \%$ oxygen followed by a ten-minute disconnection from the ventilator was deemed sufficient (20 of 71 guidelines). There was no evidence that deficient criteria for apnea testing was a result of failure to obtain timely arterial blood gases or a general reluctance to do the test. In Central and South American countries, it was noticeable that a large proportion of patients were either examined with disconnection from the ventilator only, or criteria or guidelines for the apnea test were not stated. This is potentially concerning because apnea can only be determined after introducing acute hypocarbia to induce maximal stimulation of the respiratory centres. A ten-minute period of disconnection from the ventilator in a patient with baseline hypocarbia (not uncommon when induced hyperventilation is applied to treat increased intracranial pressure) could potentially result in apnea, with $\mathrm{PaCO}_{2}$ failing to reach a target value.

The number of physicians required to diagnose brain death varies significantly throughout the world. In $44 \%$ of countries, one physician is required (including Canada); in 34\%, two physicians; and in $16 \%$ of

From the Division of Critical Care Neurology, Mayo Clinic College of Medicine, Rochester, Minnesota, USA.

Address correspondence to: Dr. Eelco F.M. Wijdicks, Mayo Clinic College of Medicine, Department of Neurology, W8B, Division of Neurological Intensive Care, 200 First Street SW, Rochester, MN 55905, USA. Phone: 507-538-1036; Fax: 507-266-4419;

E-mail: wijde@mayo.edu 
countries, more than two physicians are required. ${ }^{3}$ In other jurisdictions, the number of physicians required to diagnose brain death is unknown. Further results of this international survey showed that confirmatory tests were required in $40 \%$ of 80 nations reporting, whereas the complexity of criteria did not seem to be influenced by cultural differences. ${ }^{3}$ There were no consistent differences between Eastern and Western countries, although in some countries, an academic grade was needed to perform the apnea test (associate professor level).

The type and need of confirmatory tests vary considerably from one country to another. The choice of confirmatory tests seems to be arbitrary, with Sweden being a notable exception. In Sweden, a cerebral angiogram has to be performed twice, with an adequate period of observation in between, documenting an absence of blood flow to the brain. Although the electroencephalogram is commonly recommended worldwide, in this issue Young et al. ${ }^{4}$ make compelling arguments against its use, in favour of tests that demonstrate the absence of intracerebral blood flow.

\section{Remaining concerns}

It is apparent that for at least half of the surveyed nations in the world, and several jurisdictions in the United States, confirmation of brain death examination by a second physician is required. There is no data to support this practice, nor is there a suggestion for a requirement of more than two physicians. Requiring two physicians to determine brain death may lead to logistical problems, but in most modern intensive care units, this is achievable. Absent respiratory drive remains essential in the diagnosis of brain death. There is insufficient evidence to suggest that formal apnea testing can be deferred.

In many developed countries, prevailing cultural and religious attitudes support the clinical determination of brain death. There is no evidence to suggest that the variability in international guidelines (such as multiple observations, multiple confirmatory tests, with multiple physicians) is related to these attitudes. More strict criteria were present when donation was considered, and this is a common qualifier in many guidelines throughout the world.

Despite national recommendations, significant variability continues to exist among adult hospital policies in the United States, ${ }^{5}$ as similarly documented in Canadian adult and pediatric centres by Hornby et al. ${ }^{6}$ in this issue. It is likely that exaggerated differences would be found throughout the world if different hospital policies within each country were surveyed. There is also a lingering concern regarding the accu- racy of brain death documentation. A study by Wang et $a l^{7}$ from the University of California examined patients declared brain dead at Los Angeles County General Hospital, and found major problems with chart documentation. Corneal reflex was not documented in $43 \%$ of the cases, and motor examination was not documented in $34 \%$ of the patients. It remains unclear whether this is truly a problem of documentation or a lapse in performing a clinical examination of brain death. Organ donation procurement organizations may play an important role in checking these examinations.

When reviewing the complex guidelines of brain death determination and preparation for organ donation, one can only conclude that a consensus is needed. The intensive care unit physician survey by Doig $e t$ $a l .^{8}$ confirms this need in the Canadian context. This would require a task force that reviews the data and provides evidentiary tables. The Canadian Council for the Donation and Transplantation has admirably taken the lead with their development of national guidelines for practice and documentation. ${ }^{9}$ Acceptance of uniform criteria of brain death would then lead to a more uniform policy for brain death determination and consistent documentation. There is no satisfactory answer to the initial question posed in the title of this paper. Physicians from different countries have come to their own judgment in how to solidify the criteria. It must be driven by a concern regarding the accurate assessment of fatally injured patients. However, more physicians and more confirmatory tests cannot solve this problem. What is required is standardization of policy, appropriate education of staff, introduction of checklists in intensive care units, and brain death examination by designated, experienced physicians who have documented proficiency in brain death examination.

\section{Les critères cliniques de mort encéphalique à travers le monde : pour- quoi en arriver là ?}

L'ajout des critères neurologiques aux critères cardiorespiratoires de mort - mieux connue comme mort encéphalique - a été un changement de paradigme 
évoluant avec la possibilité de réanimer dans les salles d'urgence et les unités de soins intensifs les patients atteints de lésion cérébrale aiguë. Dans ce contexte, la réanimation a mené à la progression de l'œdème cérébral dévastateur, au déplacement et, finalement, à l'augmentation massive de la pression intracrânienne pouvant bloquer le débit sanguin à l'entrée de la base du crâne. De nombreux symposiums organisés en Suède, au Royaume-Uni et en Australie ont tenté de formuler ce qu'est la mort encéphalique fondée sur des critères neurologiques. La constatation de la mort encéphalique, dont le concept est pleinement accepté, est devenue un préalable nécessaire au don d'organe. Malgré l'acceptation généralisée des critères, une grande variabilité demeure sur la façon de les codifier à travers le monde. Une revue historique des critères de mort neurologique est présentée par Baron et coll. dans le présent numéro du Journal. ${ }^{1}$

\section{La référence}

Un article faisant autorité sur la détermination de la mort encéphalique a été publié par le Harvard ad hoc Committee. ${ }^{2}$ Dans les années 1960, on voulait produire un document, court mais complet, étant donné le besoin pressant de lignes directrices dans les unités de soins intensifs. Jusqu'à aujourd'hui, les critères de Harvard demeurent un exemple de simplicité :

1. Absence de réceptivité et de réaction.

2. Absence de mouvement et de respiration.

3. Absence de réflexes du tronc cérébral.

4. Électroencéphalogramme plat.

5. Aucune modification des résultats de tous les tests répétés $24 \mathrm{~h}$ plus tard et exclusion d'hypothermie (température corporelle $<90{ }^{\circ} \mathrm{F}$ ou $32,2{ }^{\circ} \mathrm{C}$ ) ou de dépresseurs du système nerveux central.

Les critères de mort encéphalique dans le monde Une récente enquête a exploré les pratiques internationales de diagnostic de mort encéphalique. ${ }^{3}$ Des documents originaux sur le sujet ont été obtenus de 80 pays. Les différences de critères étaient surprenantes. Alors qu'il n'y avait pas de différence quand on comparait les méthodes d'examen des réflexes du tronc cérébral, elles étaient marquées pour le test d'apnée. Ce test a toujours préoccupé les médecins, même si sa technique est simple et sans complications en général. Tester la réaction avec l'hypercapnie provoquée devrait rester la norme si notre intention est de documenter la destruction totale du tronc cérébral. La confirmation de l'apnée avec une valeur cible de $\mathrm{PaCO}_{2}$ a été utilisée dans seulement $59 \%$ de tous les documents. Dans 20 cas sur 71 , la préoxygénation avec de l'oxygène à $100 \%$, suivie d'une interruption de dix minutes de la ventilation, a été jugée suffisante. On ne pouvait savoir si la déficience du test d'apnée était le résultat d'un échec à obtenir la gazométrie du sang artériel en temps opportun ou une résistance générale à faire le test. Dans les pays d'Amérique Centrale et du Sud, il était visible qu'une grande partie des patients était examinée avec interruption de la ventilation seulement, ou les critères ou les lignes de conduite pour le test d'apnée n'étaient pas déclarées. C'est assez inquiétant, car l'apnée ne peut être déterminée qu'après avoir introduit une hypocapnie pour induire une stimulation maximale des centres respiratoires. Une interruption de dix minutes de la ventilation chez un patient qui avait une hypocapnie initiale (fait inusité quand l'hyperventilation induite est appliquée pour traiter l'hypertension intracrânienne) peut potentiellement provoquer de l'apnée avec une $\mathrm{PaCO}_{2}$ qui ne peut atteindre une valeur cible.

Le nombre de médecins nécessaires pour diagnostiquer la mort encéphalique varie significativement à travers le monde. Dans $44 \%$ des pays, un médecin est nécessaire (y compris au Canada); dans $34 \%$, il en faut deux et dans $16 \%$, plus de deux. ${ }^{3}$ Dans d'autres pays, le nombre de médecins est inconnu. D'autres résultats de cette enquête internationale ont montré que des tests de confirmation étaient nécessaires dans $40 \%$ des 80 pays répondants, tandis que la complexité des critères ne semblait pas être influencée par les différences culturelles. ${ }^{3}$ Il n'y avait pas de différences consistantes entre les pays de l'Est et de l'Ouest, bien que dans certains pays, une qualification académique était requise pour réaliser le test d'apnée (professeur agrégé).

Le type et la nécessité des tests de confirmation varient considérablement d'un pays à l'autre. Le choix de ces tests semble arbitraire, la Suède étant une exception notable. En Suède, un angiogramme cérébral doit être fait deux fois, entrecoupées d'une période suffisante d'observation pour documenter l'absence de débit sanguin vers le cerveau. Quoique l'électroencéphalogramme soit habituellement recommandé à l'échelle mondiale, dans le présent numéro Young et coll. ${ }^{4}$ présentent des arguments probants contre son usage, en faveur de tests qui démontrent l'absence de débit sanguin intracérébral.

\section{Préoccupations restantes}

Il semble que pour au moins la moitié des pays sondés, et quelques États américains, la confirmation de l'évaluation de la mort encéphalique par un second médecin soit requise. Il n'y a pas de données pour appuyer cette pratique, ni de suggestion de l'exigence 
d'avoir plus de deux médecins. La nécessité de deux médecins pour déterminer la mort encéphalique peut créer des problèmes logistiques, mais dans la plupart des unités de soins intensifs modernes, c'est faisable. L'absence de pulsion respiratoire demeure essentielle dans le diagnostic de mort encéphalique. La preuve est insuffisante pour avancer qu'un test formel d'apnée pourrait être différé.

Dans de nombreux pays développés, les croyances culturelles et religieuses dominantes appuient la détermination clinique de mort encéphalique. Il n'y a pas de preuve suggérant que la variabilité des directives internationales (comme de multiples observations, différents tests de confirmation, plus d'un médecins) soit en lien avec ces attitudes. Des critères plus stricts étaient présents quand le don d'organe était considéré et c'est un déterminant fréquent dans de nombreux guides de conduite dans le monde.

Malgré des recommandations nationales, une variabilité significative persiste dans les politiques hospitalières pour adultes aux États-Unis, ${ }^{5}$ comme dans des centres canadiens pour adultes et enfants étudiés de façon similaire par Hornby et coll. ${ }^{6}$ dans le présent numéro. Il est probable que des différences excessives seraient trouvées à travers le monde si les multiples politiques hospitalières de chaque pays étaient étudiées. Il y a aussi une inquiétude persistante quant à l'exactitude de la documentation de la mort encéphalique. Une étude de Wang et coll. ${ }^{7}$ de l'Université de Californie a vérifié les dossiers de patients pour qui une mort encéphalique avait été déclarée au Los Angeles County General Hospital et ils ont trouvé des problèmes majeurs de contenu des dossiers. Le réflexe cornéen n'était pas documenté dans $43 \%$ des cas et l'examen moteur non documenté chez $34 \%$ des patients. Il n'est pas clair si c'était là un véritable problème de documentation ou un retard à réaliser un examen clinique de la mort encéphalique. Les services d'approvisionnement en organes peuvent jouer un rôle important en vérifiant ces examens.

En passant en revue les recommandations complexes sur la détermination de la mort encéphalique et la préparation au don d'organe, on peut seulement conclure qu'un consensus est nécessaire. L'enquête menée auprès des médecins des unités de soins intensifs par Doig et coll. ${ }^{8}$ confirme cette nécessité dans le contexte canadien. Il faudra une équipe spéciale pour revoir les données et fournir des tableaux probants. Le Conseil canadien pour le don et la transplantation a admirablement pris l'initiative en élaborant des normes nationales de pratique et de documentation. ${ }^{9}$ Une acceptation de critères uniformes de mort encéphalique conduira ensuite à une politique plus uni- forme sur la détermination de la mort encéphalique et sur une documentation régulièrement obtenue. Il n'y a pas de réponse satisfaisant à la question initiale du titre de l'article. Les médecins de différents pays ont leur propre idée sur la façon de solidifier les critères. Ce doit être guidé par le souci d'une évaluation exacte des patients mortellement blessés. Cependant, plus de médecins et plus de tests de confirmation ne peuvent régler le problème. Il faut une normalisation de la politique, une formation appropriée du personnel, l'introduction de liste récapitulative dans les unités de soins intensifs et l'examen de la mort encéphalique par des médecins désignés et expérimentés qui ont une compétence reconnue pour la vérifier.

\section{References}

1 Baron L, Shemie SD, Teitelbaum J, Doig CJ. Brief review: History, concept and controversies in the neurological determination of death. Can J Anesth 2006; 53: 602-9.

2 Anonymous. A definition of irreversible coma. Report of the Ad Hoc Committee of the Harvard Medical School to Examine the Definition of Brain Death. JAMA 1968; 205: 337-40.

3 Wijdicks EF. Brain death worldwide: accepted fact but no global consensus in diagnostic criteria. Neurology 2002; 58: 20-5.

4 Young B, Shemie SD, Doig CJ, Teitelbaum J. The role of ancillary tests in the neurological determination of death. Can J Anesth 2006; 53: 620-7.

5 Powner DJ, Hernandez M, Rives TE. Variability among hospital policies for determining brain death in adults. Crit Care Med 2004; 32: 1284-8.

6 Hornby K, Shemie SD, Teitelbaum J, Doig C. Variability in hospital-based brain death guidelines in Canada. Can J Anesth 2006; 53: 613-19.

7 Wang MY, Wallace P, Gruen JP. Brain death documentation: analysis and issues. Neurosurgery 2002; 51 : 731-5; discussion 735-6.

8 Doig CJ, Young K, Teitelbaum J, Shemie SD. Brief survey: Determining brain death in Canadian intensive care units. Can J Anesth 2006; 53: 609-12.

9 Shemie SD, Doig C, Dickens B, et al. Severe brain injury to neurological determination of death: Canadian forum recommendations. CMAJ 2006; 174: S1-13. 